\title{
Sandpile models and random walkers on finite lattices
}

\author{
Yehiel Shilo and Ofer Biham \\ Racah Institute of Physics, The Hebrew University, Jerusalem 91904, Israel
}

\begin{abstract}
Abelian sandpile models, both deterministic, such as the Bak, Tang, Wiesenfeld (BTW) model [P. Bak, C. Tang and K. Wiesenfeld, Phys. Rev. Lett. 59, 381 (1987)], and stochastic, such as the Manna model [S.S. Manna, J. Phys. A 24, L363 (1991)], are studied on finite square lattices with open boundaries. The avalanche size distribution $P_{L}(n)$ is calculated for a range of system sizes, $L$. The first few moments of this distribution are evaluated numerically and their dependence on the system size is examined. The sandpile models are conservative in the sense that grains are conserved in the bulk and can leave the system only through the boundaries. It is shown that the conservation law provides an interesting connection between sandpile models and random walk models. Using this connection, it is shown that the average avalanche sizes, $\langle n\rangle_{L}$, for the BTW and the Manna models are equal to each other, and both are equal to the average path-length of a random walker starting from a random initial site on the same lattice of size $L$. This is in spite of the fact that sandpile models with deterministic (BTW) and stochastic (Manna) toppling rules exhibit different critical exponents, indicating that they belong to different universality classes.
\end{abstract}




\section{INTRODUCTION}

Sandpile models have been studied extensively in the past fifteen years as a paradigm of self organized criticality (SOC) [1, 2, 3]. SOC provides a useful framework for the analysis of driven nonequilibrium systems which dynamically evolve into a critical state. At the critical state these systems exhibit avalanche dynamics with long-range spatial and temporal correlations, which resembles the behavior at equilibrium critical points. In sandpile models, defined on a lattice, grains are deposited randomly until the height at some site exceeds the threshold, thus becoming unstable. The unstable site is toppled and grains are distributed between its nearest neighbors, which may become unstable too, resulting in an avalanche. These models were found to be self-driven into a critical state in which the avalanche sizes follow a power-law distribution. The critical state, which can be characterized by various critical exponents and scaling functions, was studied using both theoretical 4, 5, 6, 7, 8, 9, 10, 11] and numerical approaches [12, 13, 14, 15, 16, 17, 18, 19, 20]. These studies stimulated an effort to examine the utility of the SOC framework to the understanding of empirical phenomena such as earthquakes avalanches in granular flow and mass extinctions [21].

To examine the dependence of the critical state on various properties of the models, different sandpile models have been introduced. These include the stochastic model introduced by Manna [22]. The issue of universality has been studied. Analytical studies [23, 24] and numerical simulations [25] indicated that the Manna model, which is stochastic, belongs to the universality class of the original model introduced by Bak, Tang and Wiesenfeld (BTW) which is deterministic (namely, has a deterministic toppling rule). However, numerical simulations using an extended set of critical exponents showed that deterministic and stochastic models exhibit different scaling properties and thus belong to different universality classes [26, 27, 28]. Further support for this result was obtained using multifractal analysis [29],

moment analysis [30] as well as studies of sandpile models as closed systems [31, 32, 33]. The crossover between the two classes was also studied [34]. In the case of directed models it was shown analytically that deterministic and stochastic models belong to different universality classes [4, 35, 36].

In this paper we present a connection between abelian sandpile models and random walkers on finite lattices, which is a result of the conservation laws. In the sandpile models each avalanche starts with the addition of one grain. The models are conservative in the 
sense that grains are conserved in the bulk of the system and can leave it only through the boundaries. It is thus clear that under steady state conditions, the average number of grains leaving the system per avalanche is also one. Here, the avalanche size is defined as the number of hops of grains that are toppled from unstable sites during an avalanche. The avalanche size is thus equal to the number of toppling events (or relaxations) of unstable sites during the avalanche times the number of grains that topple in each event of this type. We show that in both the BTW and the Manna models, each grain moves like a random walker, starting at the site in which it was deposited, until it falls off the boundaries. Using these features we show that the average avalanche size is the same for the two models. Moreover, it is equal to the average path length of a random walker starting from a random site on the same lattice, until it falls off the edge.

In order to demonstrate these properties we examine the avalanche size distribution of the Abelian sandpile models and the distribution of path length of a random walker on finite square lattices. The average path length of a random walker on a lattice of size $L$ is calculated exactly using a method proposed by Walsh and Kozak [37, 38]. The entire distribution of the path lengths of random walkers starting at random sites on the finite

lattice is also calculated using a related method proposed by Soler [39]. The results are also compared to direct numerical simulation of the random walk. The avalanche size distribution of the Abelian sandpile models is obtained from direct numerical simulations as well as from an exact formula introduced by Dhar [6] .

The paper is organized as follows. The sandpile models are described in Sec. II. The distributions of path lengths of random walkers on finite lattices and their averages are studied in Sec. III. The connection between sandpile models and random walkers is examined in Sec. IV. The simulations and results are given in Sec. V, followed by a discussion in Sec. VI and a summery in Sec. VII.

\section{SANDPILE MODELS}

Consider a sandpile model on a $d$-dimensional cubic lattice of linear size $L$. Each site $\mathbf{i}$ is assigned a dynamic variable $E(\mathbf{i})$ which represents some physical quantity such as energy, grain density, stress, etc. A configuration $\{E(\mathbf{i})\}$ is called stable if for all sites $E(\mathbf{i})<E_{c}$, where $E_{c}$ is a threshold value. The evolution between stable configurations is by the following 
rules: (i) Adding energy. Given a stable configuration $\{E(\mathbf{j})\}$ we select a site $\mathbf{i}$ at random and increase $E(\mathbf{i})$ by some amount $\delta E$. When an unstable configuration is reached rule (ii) is applied. (ii) Relaxation (or toppling) rule. If $E(\mathbf{i}) \geq E_{c}$, relaxation takes place and energy is distributed in the following way:

$$
\begin{aligned}
E(\mathbf{i}) & \rightarrow E(\mathbf{i})-\sum_{\mathbf{e}} \Delta E(\mathbf{e}) \\
E(\mathbf{i}+\mathbf{e}) & \rightarrow E(\mathbf{i}+\mathbf{e})+\Delta E(\mathbf{e})
\end{aligned}
$$

where $\mathbf{e}$ are a set of vectors from the site $\mathbf{i}$ to some neighbors. As a result of the relaxation, $E(\mathbf{i}+\mathbf{e})$ for one or more of the neighbors may reach or exceed the threshold $E_{c}$. The relaxation rule is then applied until a stable configuration is obtained. The resulting sequence of topplings is an avalanche which propagates through the lattice.

Avalanches can be characterized by their size. The size $s$ of an avalanche is the total number of toppling events that occurred during the course of the avalanche. In the models studied here the number of grains that topple from an unstable site is $E_{c}=\sum_{\mathbf{e}} \Delta E(\mathbf{e})$. Throughout the rest of the paper we will denote the avalanche size by

$$
n=E_{c} \cdot s,
$$

namely, by the number of hops of individual grains that take place during the avalanche. This will allow us to consider models with different values of $E_{c}$ on a common footing.

The avalanche size distribution is denoted by $P_{L}(n), n=0,1, \ldots$, namely, the probability of a randomly chosen avalanche to be of size $n$. The normalization condition is given by

$$
\sum_{n=0}^{\infty} P_{L}(n)=1 .
$$

Numerical simulations show that the avalanche size distribution for a lattice of size $L$ has the power-law form:

$$
P_{L}(n) \sim n^{-\tau_{L}}, \quad n=1,2, \ldots
$$

For some of the models the results for $\tau_{L}$ exhibit a significant dependence on the system size. The critical exponents $\tau_{L}$ turn out to depend on the vector $\Delta E$ to be termed relaxation 
vector. For a square lattice with relaxation to nearest neighbors it is of the form $\Delta E=$ $\left(E_{N}, E_{E}, E_{S}, E_{W}\right)$, where $E_{N}, E_{E}, E_{S}$ and $E_{W}$ are the amounts transferred to the northern, eastern, southern and western nearest neighbors respectively. The average avalanche size on a lattice of size $L$ is given by

$$
\langle n\rangle_{L}=\sum_{n=0}^{\infty} n P_{L}(n) .
$$

The sandpile models considered in this paper are conservative in the sense that the grains are conserved in the bulk and can leave the system only through the open boundaries. When an avalanche reaches a boundary site, some energy is transferred out of the system (namely, dissipation takes place at the boundaries). The critical state is reached spontaneously in the limit in which the random addition of energy (or drive) is infinitely slow (practically it means that the next energy unit is added only after the previous avalanche is completed). This state is characterized by a power-law distribution of avalanche sizes [Eq. (4)]. In the critical state the added energy $\delta E$ per avalanche, is balanced, on average, by the energy that flows out through the boundaries. Therefore, the average amount of energy leaving the system per avalanche is $\delta E$.

In the BTW model, $E_{c}=4, \delta E=1$ and $\Delta E=(1,1,1,1)$. Since $\Delta E$ is a constant, this model is clearly deterministic. Note that since $\Delta E$ is independent of $E(\mathbf{i})$, if an active site with $E(\mathbf{i})>E_{c}$ is toppled, it remains non-empty after the toppling event had occurred. A useful way to analyze the BTW model is by its toppling matrix $\Delta$, which for an $L \times L$ lattice is a matrix of size $L^{2} \times L^{2}$. Consider a pair of sites $\mathbf{i}=\left(i_{x}, i_{y}\right), \mathbf{j}=\left(j_{x}, j_{y}\right)$ and denote $i=L i_{x}+i_{y}, j=L j_{x}+j_{y}$ where $0 \leq i_{x}, i_{y}, j_{x}, j_{y} \leq L-1$. The matrix element $\Delta_{i, j}$ where $i \neq j$ is the number of grains given to site $\mathbf{j}$ when site $\mathbf{i}$ topples (up to a minus sign). The number of grains leaving site $\mathbf{i}$ in such event is given by the diagonal element $\Delta_{i, i}$. Therefore, the toppling matrix is :

$$
\Delta_{i, j}= \begin{cases}4 & \mathbf{i}=\mathbf{j} \\ -1 & \mathbf{i} \text { and } \mathbf{j} \text { are nearest neighbor sites } \\ 0 & \text { otherwise. }\end{cases}
$$

Consider the toppling of a given site i. It can be described by: 


$$
E(\mathbf{j}) \rightarrow E(\mathbf{j})-\Delta_{i j}
$$

for all sites $\mathbf{j}$.

In the class of stochastic sandpile models, introduced by Manna, a set of neighbors is randomly chosen for relaxation [22] once a site becomes unstable. Such models can be specified by a set of relaxation vectors, each vector is assigned with a probability for its appearance. There are several models in this class. One of them is a two-state model with $E_{c}=2$ and two relaxation vectors $(1,0,1,0)$ and $(0,1,0,1)$ each one applied with a probability of $1 / 2[40]$. Another two-state model includes six relaxation vectors, namely $(1,1,0,0)$, $(1,0,0,1),(0,1,1,0),(0,0,1,1),(1,0,1,0)$ and $(0,1,0,1)$, each one applied with a probability of $1 / 6$ [26]. In this paper we consider a two-state model in which each of the two grains of an unstable site is toppled randomly to one of the four neighbors (with probability $1 / 4$ to each direction). There is no correlation between the directions picked for these two grains. The set of relaxation vectors includes all the 10 possible vectors $\Delta E$ of integer components for which $E_{N}+E_{E}+E_{W}+E_{S}=2$. Each of the six vectors of the previous model appears with probability $2 / 16$, while each of the four vectors: $(2,0,0,0),(0,2,0,0),(0,0,2,0)$ and $(0,0,0,2)$, appears with probability $1 / 16$. This model will be called the unrestricted two-state Manna model.

The average avalanche size $\langle n\rangle$ for the BTW model on a lattice of size $L$ was calculated exactly by Dhar [6]. He showed that the matrix element $\Delta_{i, j}^{-1}$ represents the average number of toppling events taking place at site $\mathbf{j}$ when a grain starts an avalanche after being deposited at site $\mathbf{i}$. By summing all the elements of $\Delta^{-1}$, using the eigenvectors and eigenvalues of $\Delta$ [41] it was found that

$$
\langle n\rangle=\frac{1}{L^{2}(L+1)^{2}} \sum_{k, l} \frac{\cot ^{2} \theta_{k} \cot ^{2} \theta_{l}}{\sin ^{2} \theta_{k}+\sin ^{2} \theta_{l}},
$$

where

$$
\theta_{m}=\frac{\pi m}{2(L+1)}
$$

for any integer $m$, and the summation over $k, l$ is over all odd integers $1 \leq k, l \leq L$. The dependence of $\langle n\rangle$ on the system size was found to be $\langle n\rangle \sim L^{2}$. This analysis was recently 
extended to dissipative abelian models [42].

\section{RANDOM WALKS ON FINITE LATTICES}

Consider a random walker on a square lattice of size $L$. The walker starts at a random initial site $\mathbf{i}=\left(i_{x}, i_{y}\right)$, where $0 \leq i_{x}, i_{y} \leq L-1$. At each step the walker has four possible moves, to one of the sites $\mathbf{i}_{\mathbf{R}}=\left(i_{x}+1, i_{y}\right), \mathbf{i}_{\mathbf{L}}=\left(i_{x}-1, i_{y}\right), \mathbf{i}_{\mathbf{U}}=\left(i_{x}, i_{y}+1\right)$ and $\mathbf{i}_{\mathbf{D}}=\left(i_{x}, i_{y}-1\right)$, each picked with equal probability. The boundaries are open, and thus a random walker starting at any site $\left(i_{x}, i_{y}\right)$ will eventually fall off the edge [37, 38]. The number of moves it will make depends on the location of the initial site as well as on the particular realization of the random moves generating the path of the given walker. Therefore, there is a probability distribution $p_{\mathbf{i}}(n)$, where $n=1,2 \ldots, \infty$, that a random walker starting at site $\left(i_{x}, i_{y}\right)$ will fall off the edge after $n$ moves. We will first calculate the average of this distribution given by

$$
\left\langle n_{\mathbf{i}}\right\rangle=\sum_{n=1}^{\infty} n \cdot p_{\mathbf{i}}(n) .
$$

The boundary conditions are given by $\left\langle n_{\mathbf{i}}\right\rangle=0$ for sites beyond the edge of the $L \times L$ lattice, namely those for which $i_{x}=-1$ or $L$, or $i_{y}=-1$ or $L$. Since on its way to the boundaries the walker must pass through at least one of the nearest neighbors of the site $\mathbf{i}$, there is a relation between $\left\langle n_{\mathbf{i}}\right\rangle$ and the corresponding averages for its nearest neighbors of the form

$$
\left\langle n_{\mathbf{i}}\right\rangle=\frac{1}{4}\left(\left\langle n_{\mathbf{i}_{\mathbf{R}}}\right\rangle+\left\langle n_{\mathbf{i}_{\mathbf{L}}}\right\rangle+\left\langle n_{\mathbf{i}_{\mathbf{D}}}\right\rangle+\left\langle n_{\mathbf{i}_{\mathbf{U}}}\right\rangle\right)+1 .
$$

This set of $L^{2}$ coupled linear equations can be written in a matrix form as

$$
\frac{1}{4} \Delta\langle\mathbf{n}\rangle=\mathbf{1}
$$

where the matrix $\Delta$ is identical to the toppling matrix of the BTW model, given in Eq. (6). The vector $\langle\mathbf{n}\rangle$, consists of the $L^{2}$ components $\langle n\rangle_{i}=\left\langle n_{\mathbf{i}}\right\rangle$, where $\mathbf{i}=\left(i_{x}, i_{y}\right)$ and $i=L i_{x}+i_{y}$ $\left(i=0,1, \ldots, L^{2}-1\right)$. The $L^{2}$-dimensional vector $\mathbf{1}$ is given by $\mathbf{1}=(1,1, \ldots, 1)$.

In order to reduce the number of equations we shall use the symmetry properties of the square lattice, that has one horizontal, one vertical and two diagonal reflection axes. Any two sites with the same symmetry properties are called sites of the same "type". Due to the symmetry it is sufficient to examine the sites in the triangle bounded by the vertical 
axis from the center upwards and by the diagonal axis from the center to the upper - right corner (Fig. 1). In this triangle there is one site of each type. The number of sites in the triangle is

$$
N=\frac{(L+1)(L+3)}{8}
$$

when $L$ is odd, and

$$
N=\frac{L(L+2)}{8}
$$

when $L$ is even. The $\mathrm{N}$ linear equations for $\langle n\rangle_{i}$ are of the form :

$$
\frac{1}{4} D\langle\mathbf{n}\rangle=\mathbf{1}
$$

where $\mathrm{D}$ is an $N \times N$ matrix. The matrix elements of $\mathrm{D}$ are:

$$
D_{i, j}= \begin{cases}4-f(i, i) & i=j \\ -f(i, j) & i \neq j\end{cases}
$$

where $f(i, j)$ is the number of sites of type $j$ that are nearest neighbors to a site of type $i$. For $L=3 \mathrm{Eq}$. (15) takes the form

$$
\left(\begin{array}{ccc}
1 & -1 & 0 \\
-\frac{1}{4} & 1 & -\frac{1}{2} \\
0 & -\frac{1}{2} & 1
\end{array}\right)\left(\begin{array}{c}
\langle n\rangle_{1} \\
\langle n\rangle_{2} \\
\langle n\rangle_{3}
\end{array}\right)=\left(\begin{array}{l}
1 \\
1 \\
1
\end{array}\right)
$$

and its solution can be easily found to be $\langle\mathbf{n}\rangle=(4.5,3.5,2.75)$. Now, having explicit values for the $\langle n\rangle_{i}$ 's, the average path length $\langle n\rangle$ of a random walker that starts at a random site on the $3 \times 3$ lattice is

$$
\langle n\rangle=\frac{\left\langle n_{1}\right\rangle+4\left\langle n_{2}\right\rangle+4\left\langle n_{3}\right\rangle}{9}=3.277 .
$$

The probability distribution $p_{\mathbf{i}}(n)$ of a walker starting at site $\mathbf{i}$ to fall off the edge after $n$ moves can also be calculated [39]. One can then average $p_{\mathbf{i}}(n)$ over all lattice sites and obtain the probability distribution $P_{L}(n), n=1,2, \ldots$, that a walker starting at a random site on the lattice will fall off the edge after $n$ steps. This probability is given by 


$$
P_{L}(n)=\frac{1}{L^{2}} \sum_{\mathbf{i}} p_{\mathbf{i}}(n) .
$$

Note that for the random walk model, $P_{L}(n)$ is defined only for $n \geq 1$, because the random walker must make at least one move in order to fall off the edge. The normalization condition will thus take the form

$$
\sum_{n=1}^{\infty} P_{L}(n)=1
$$

The moments of this distribution are given by

$$
\left\langle n^{q}\right\rangle_{L}=\sum_{n=1}^{\infty} n^{q} \cdot P_{L}(n)
$$

The average path length (first moment) is given by Eq. (21) with $q=1$, the second moment by $q=2$ and so on. The calculation of $p_{\mathbf{i}}(n)$ is done recursively starting from the boundaries. The probability that a walker starting at site $\mathbf{i}$ will fall off the edge after $n$ steps is given by

$$
p_{\mathbf{i}}(n)=\frac{1}{4}\left[p_{\mathbf{i}_{\mathbf{R}}}(n-1)+p_{\mathbf{i}_{\mathbf{L}}}(n-1)+p_{\mathbf{i}_{\mathbf{U}}}(n-1)+p_{\mathbf{i}_{\mathbf{D}}}(n-1)\right],
$$

namely, it is the average over the four nearest neighbors, of the probabilities that a walker starting in one of them will fall off the edge after $n-1$ steps. The boundary conditions are $p_{\mathbf{i}}(n)=0$ where $i_{x}=-1, L$ or $i_{y}=-1, L$ and $n=1,2, \ldots, \infty$, reflecting the fact that these indices represent sites that are already over the edge. The initial conditions for the recursive procedure for the calculation of $p_{\mathbf{i}}(n), n=1,2, \ldots$, are given by $p_{\mathbf{i}}(1)=1 / 4$ for all the edge sites, except for the corner sites, for which $p_{\mathbf{i}}(1)=1 / 2$. For all other sites $p_{\mathbf{i}}(1)=0$. The distribution $p_{\mathbf{i}}(n), n=2, \ldots$, is calculated recursively using Eq. (22) for all sites, starting at $n=2$ and increasing $n$ by 1 after each scan of the lattice. The average path length $\langle n\rangle$ of a random walker starting at a random site is then obtained from Eqs. (19) and (21).

\section{THE CONNECTION BETWEEN SANDPILE MODELS AND RANDOM WALK MODELS}

Consider a grain deposited at a random site in the unrestricted two-state Manna model. It will initiate a small or a large avalanche and will typically stay on the lattice for many 
subsequent avalanches until it will fall off the edge. Most of this time the grain is alone in its site. Whenever it will share the site with another grain, both of them will topple randomly (and independently) to nearest neighbor sites. The path of the grain on the lattice is, in fact, a random walk since there is no correlation between one move to another 43]. This path starts at the site into which the grain was deposited and ends at the edge site through which it leaves the system. The different walkers are uncorrelated since the directions chosen by the two grains that topple from an unstable site are independent. The only correlation is between the times that different walkers make their random moves. This temporal correlation appears because two walkers need to occupy the same site in order to move.

This property, that the path of each grain in the sandpile model is a random walker, is not limited to the unrestricted two-state Manna model. It is a general property of the conservative-Abelian models, in which the grains are discrete entities. It is thus a common property of models that belong to both the deterministic and the stochastic universality classes. Consider, for example, the BTW model. It is convenient to consider the grains as distinguishable particles by naming each one of them according to the running number representing the order of their deposition into the system. When an unstable site topples, we will pick the grain that entered this site first, and choose randomly one of the four directions $(N, E, S, W)$ for it to move. For the second grain we will choose randomly among the three remaining directions, and so on for the third and fourth grains. It is clear that there is no correlation between the directions of consecutive moves of each grain and no bias. Therefore, each grain follows a path of a random walker. Unlike the unrestricted two-state Manna model, in the BTW model there is correlation between the directions of different grains that topple from the same site, since they cannot move in the same direction.

Each avalanche in the sandpile models starts with a new grain deposited randomly. Therefore, on average each avalanche drops one grain off the edge. Since the grains follow random walker paths from the random initial site to an edge site, the average number of hops that take place in a single avalanche must be equal to the average number of steps, that is required for a random walker deposited randomly on the lattice to reach the edge. We thus conclude that the average avalanche size of a sandpile model on a lattice of size $L$ is the same for the BTW and Manna models, and equal to the average path length of a random walker deposited randomly on the same lattice. 


\section{SIMULATIONS AND RESULTS}

To examine the connection between the random walk model and the sandpile models on finite lattices, we have performed numerical simulations of both systems. Direct simulations of the random walk model were performed on a square lattice of size $L$ with open boundaries. In each run the walker started at a random site on the lattice. The random walk path was generated until the walker fell off the edge. The path length, namely the number, $n$, of moves it made from the initial site to the edge was recorded. From this data, the distribution of path lengths $P_{L}(n)$ was generated and its average $\langle n\rangle_{L}$ was calculated. The average $\langle n\rangle_{L}$ was also calculated using the Walsh-Kozak method [37, 38]. The distribution $P_{L}(n)$ was also calculated using the Soler method [39] and $\langle n\rangle_{L}$ was extracted from it. Direct simulations of the BTW and Manna models were performed, from which the avalanche size distributions were obtained. The average avalanche size for each model was calculated as a function of the lattice size $L$. The average avalanche size for the BTW model was also calculated using Dhar's formula [Eq. (8) ]. The values of $\langle n\rangle$ vs. $L$, obtained for both the random walk and sandpile models are shown in Fig. 2. They all coincide perfectly, except for some slight fluctuations in the direct simulation data for the larger values of $L$. This confirms the connection between the random walk and sandpile models. Fitting $\langle n\rangle_{L}$ vs. $L$ to a polynomial function we obtain that $\langle n\rangle_{L}=a L^{2}+b L$, where $a=0.14$ and $b=0.56$.

While the averages are found to be the same for the sandpile models and the random walk models, the distributions $P_{L}(n)$ turn out to be different. To calculate the distribution $P_{L}(n)$ of path lengths of random walkers starting from random sites on a square lattice of size $L$, we used the Soler method [39]. The calculation was done for square lattices of sizes $L=32,64,128,256$ and 512 . To obtain a scaling function we rescaled $n$ for each system size by the average path length $\langle n\rangle_{L}$. The scaling function $P\left(n /\langle n\rangle_{L}\right)=\langle n\rangle_{L} \cdot P_{L}(n)$ is shown in Fig. 3 on a double logarithmic scale. The scaling function exhibits a linear range, up to an upper cutoff around $n /\langle n\rangle_{L}=1$. The slope of the linear range turns out to be approximately $-1 / 2$. This function can be considered in the framework of first passage problems of a random walks on a finite lattice [44].

The avalanche size distributions for the BTW and Manna models were obtained from direct numerical simulations for lattice sizes $L=32,64,128,256$ and 512 . The rescaled distribution functions are shown in Fig. 4(a) for the BTW model and in Fig. 4(b) for the 
Manna model. In both cases the data collapse is not complete, due to the finite size dependence of the critical exponent $\tau_{L}$. Fitting the data for $L=512$ to Eq. (44) we obtain that $\tau_{L}=1.12 \pm 0.02$ for the BTW model and $\tau_{L}=1.27 \pm 0.02$ for the Manna model, in agreement with previous results [22, 27, 30].

The first three moments of the distribution of path-lengths of the random walk vs. $L$ are shown in In Fig. 5 . The results were obtained both by direct simulation and by calculating $P_{L}(n)$ using the Soler method [39]. Fitting these graphs to power laws in $L$ we find that $\left\langle n^{q}\right\rangle_{L} \sim L^{2 q}$ (the slopes of the best linear fits are 1.98 $\pm 0.04,3.97 \pm 0.06$, and $5.96 \pm 0.08$ for $q=1,2$ and 3, respectively).

For sandpile models, in the large system limit, the first moment of the avalanche size distribution scales like $\langle n\rangle_{L} \sim L^{2}$. Higher moments are expected to scale like

$$
\left\langle n^{q}\right\rangle_{L} \sim L^{\sigma(q)},
$$

with $\sigma(q)>2 q$ for $q>2$. The first three moments of the avalanches size distributions of the BTW and Manna models, vs. L, are shown in Fig. [6] The slopes of the best linear fits for the BTW model are $\sigma(q)=1.98 \pm 0.02,4.68 \pm 0.04$, and $7.52 \pm 0.08$ for $q=1,2$ and 3, respectively. The slopes of the best linear fits for the Manna model are $\sigma(q)=1.97 \pm 0.02$, $4.73 \pm 0.04$, and $7.48 \pm 0.08$ for $q=1,2$ and 3 , respectively. These linear fits were obtained for lattice sizes in the range $64 \leq L \leq 1024$. The results for the first moment are identical (within the error bars) for the two models, and coincide with the results for the random walk model, and thus confirm the conclusions of the analysis above. Surprisingly, the values of $\sigma(q)$ for the BTW and Manna models are approximately the same (within the error bars), also for $q=2$ and 3. This is in spite of the fact that the avalanche size distributions of the two models are characterized by different exponents $\tau_{L}$. This behavior has to do with deviations from the power-law behavior near the upper cutoffs of the distributions. The results for the higher moments are in agreement with those presented in Refs. 30, 45].

\section{DISCUSSION}

Power-law distributions were observed in a wide variety of natural systems as well as in economic systems, computer networks, linguistics and other fields. Some examples include the energy distribution between scales in turbulence [46], the distribution of earthquake 

and wealth [50, 51, 52, 53], the distribution of the number of links pointing to sites in the internet [54, 55] and the distribution of the frequency of appearance of words in texts [48]. A common feature of such system is that they consist of a large number of elementary degrees of freedom that interact with each other in a complex way. Power-law distributions typically appear when these interactions give rise to long-range correlations with no characteristic length-scale.

Consider a power-law distribution of the form

$$
P_{L}(n)=A(L) \cdot n^{-\tau_{L}}
$$

limited to the range between $n_{\min }(L)$ and $n_{\max }(L)$. For simplicity we will assume that the lower cutoff is fixed to $n_{\min }(L)=1$. As in the case of the sandpile models, we will assume that the upper cutoff is limited by the system size, $L$, and that $n_{\max }(L) \rightarrow \infty$ when $L \rightarrow \infty$. The probability distribution $P_{L}(n)$ should satisfy the normalization condition

$$
\int_{n_{\min }(L)}^{n_{\max }(L)} P_{L}(n) \mathrm{d} n=1,
$$

namely, $A(L)=\left(1-\tau_{L}\right) /\left(n_{\max }^{1-\tau_{L}}-1\right)$. In order for $A(L)$ to converge to a finite nonzero value as $L \rightarrow \infty$, the exponent $\tau_{L}$ must satisfy $\tau_{L}>1$ in the infinite system limit. The first moment of the distribution,

$$
\langle n\rangle_{L}=\int_{n_{\min }(L)}^{n_{\max }(L)} n P_{L}(n) \mathrm{d} n
$$

thus takes the form

$$
\langle n\rangle_{L}=\frac{\left(1-\tau_{L}\right)\left[n_{\max }(L)^{2-\tau_{L}}-1\right]}{\left(2-\tau_{L}\right)\left[n_{\max }(L)^{1-\tau_{L}}-1\right]} .
$$

We observe that for $\tau_{L}>2$ the first moment converges to a finite value in the infinite system limit. On the other hand, for $1<\tau_{L}<2$, the first moment, $\langle n\rangle_{L}$, diverges for $L \rightarrow \infty$. We thus obtain a connection between the behavior of the first moment of the distribution in the infinite system limit and the range of values that the exponent $\tau_{L}$ can take.

For the sandpile models studied here the exponent $\tau_{L}$ is in the range $1<\tau_{L}<2$, and indeed, the average avalanche size diverges according to $\langle n\rangle=a L^{2}$ as $L \rightarrow \infty$. The upper cutoff $n_{\max }$ can be expressed as a function of $L$ and $\tau_{L}$ : 


$$
n_{\max }=\left[\frac{a\left(2-\tau_{L}\right)}{\tau_{L}-1}\right]^{\frac{1}{2-\tau_{L}}} L^{\frac{2}{2-\tau_{L}}} .
$$

Using this upper cutoff in the calculation of higher moments we obtain that in the infinite system limit they will scale according to Eq. (23) with

$$
\sigma(q)=\frac{2\left(q+1-\tau_{L}\right)}{2-\tau_{L}}
$$

Note that Eq. (29) predicts significantly different values of $\sigma(q)$ for the BTW and the Manna models, due to the difference in the values of $\tau_{L}$ for the two models. On the other hand, Fig. [6] shows nearly identical values of $\sigma(q), q=2,3$ for the two models. The fact that these two moments coincide seems to be due to the deviations from power-law behavior near the upper-cutoffs. The effect of these deviations is significant for high moments.

Recently, multifractal scaling was observed in the avalanche size distribution of the BTW model [29, 56, 57]. This indicates that a finite size scaling analysis of the form of Eq. (24) is not sufficient for describing the scaling behavior of the BTW model, although it was found to apply in the case of the Manna model [29, 56, 57].

Exponents $\tau_{L}$ in the range $1<\tau_{L}<2$, were observed empirically in the distribution of earthquake magnitudes. Many other systems exhibit values of $\tau_{L}$ in the range $2<\tau_{L}<3$. In these systems the first moment is kept finite in the infinite system limit, while the second moment, that characterizes the fluctuations in the system diverges. Consider, for example, a directed graph model describing an internet-like network. Each node in the graph has a fixed number, $r$, of links pointing outwards to other nodes. The graph is constructed such that the probability of each node to receive links from newly added nodes is proportional to the number of incoming links that it already has. For a network that reached a size of $L$ nodes, this process generates a power-law distribution of the number of incoming links among the nodes. In the resulting network the total number of outgoing links must be equal to the total number of incoming links. Since each node has $r$ outgoing links, the average number of incoming links per node must be $\langle n\rangle_{L}=r$, independent of the size $L$ of the network. Since the first moment of the distribution is kept finite, while the second moment diverges as $L \rightarrow \infty$, the exponent $\tau_{L}$ must be in the range $2<\tau_{L}<3$ in the infinite system limit. The notable feature of the network system is that the average, $\langle n\rangle_{L}$, of the power-law distribution of the incoming links is forced to remain constant and independent of the system 
size. Systems that have this feature are common. Other examples include the distribution of the number of citations to scientific papers. Each citation is a directed link from a newer paper to an older one. While the distribution of the number of outgoing links per paper is narrow, the distribution of incoming links is broad, and resembles a power-law distribution. Another example is the distributions of income and wealths in western societies, that were found to exhibit power-law behavior, at least in the high income sectors, with exponents in the range $2<\tau_{L}<3$. Here the argument is not as easy to make. However, one may argue that the average of these distributions must be connected to the average productivity per worker. This productivity remains finite when the size of the economy increases.

\section{SUMMARY AND CONCLUSIONS}

Abelian sandpile models (both deterministic and stochastic) and random walk models have been studied on finite square lattices with open boundaries. The avalanche size distributions of the sandpile models, as well as the distributions of the lengths of the random-walk paths were calculated using various methods. It was shown that, due to the conservation laws, the averages $\langle n\rangle$ of the avalanche size distributions of the deterministic and stochastic models are the same, and that they are both equal to the average length of the random-walk paths starting from random sites on the same lattice.

\section{Acknowledgments}

We thank O. Malcai for many useful discussions. This work was supported by the USIsrael Binational Science Foundation, under Grant No. 9800097.

[1] P. Bak, C. Tang and K. Wiesenfeld, Phys. Rev. Lett. 59, 381 (1987).

[2] P. Bak, C. Tang and K. Wiesenfeld, Phys. Rev. A 38, 364 (1988).

[3] C. Tang and P. Bak, Phys. Rev. Lett. 60, 2347 (1988).

[4] D. Dhar and R. Ramaswamy, Phys. Rev. Lett. 63, 1659 (1989).

[5] T. Hwa and M. Kardar, Phys. Rev. Lett. 62, 1813 (1989).

[6] D. Dhar, Phys. Rev. Lett. 64, 1613 (1990). 
[7] J. M. Carlson, J. T. Chayes, E. R. Grannan and G. H. Swindle, Phys. Rev. Lett. 65, 2547 (1990).

[8] G. Grinstein and D.-H. Lee, Phys. Rev. Lett. 66, 177 (1991).

[9] M. Paczuski and S. Boettcher, Phys. Rev. Lett. 77, 111 (1996).

[10] C. Vanderzande and F. Daerden, Phys. Rev. E 63, 030301 (2001).

[11] A. Vespignani and S. Zapperi, Phys. Rev. E 57, 6345 (1998).

[12] L. P. Kadanoff, S. R. Nagel, L. Wu and S.-M. Zhou, Phys. Rev. A 39, 6524 (1989).

[13] P. Grassberger and S.S. Manna, J. Phys. (France) 51, 1077 (1990).

[14] S.S. Manna, J. Stat. Phys. 59, 509 (1990).

[15] K. Christensen, H.C. Fogedby, and H.J. Jensen, J. Stat. Phys. 63, 653 (1991).

[16] K. Christensen and Z. Olami, Phys. Rev. E 48, 3361 (1993).

[17] M. Paczuski and S. Boettcher, Phys. Rev. E 56, R3745 (1997).

[18] S. Lübeck and K.D. Usadel, Phys. Rev. E 55, 4095 (1997).

[19] S. Lübeck, Phys. Rev. E 56, 1590 (1997).

[20] D.V. Ktitarev, S. Lübeck, P. Grassberger and V.B. Priezzhev, Phys. Rev. E 61, 81 (2000).

[21] P. Bak, How Nature Works (Springer-Verlag, New York, 1996).

[22] S.S. Manna, J. Phys. A 24, L363 (1991).

[23] L. Pietronero, A. Vespignani and S. Zapperi, Phys. Rev. Lett. 72, 1690 (1994).

[24] A. Vespignani, S. Zapperi and L. Pietronero, Phys. Rev. E 51, 1711 (1995).

[25] A. Chessa, H.E. Stanley, A. Vespignani and S. Zapperi, Phys. Rev. E 59, R12 (1999).

[26] A. Ben-Hur and O. Biham, Phys. Rev. E 53, R1317 (1996).

[27] E. Milshtein, O. Biham and S. Solomon, Phys. Rev. E 58, 303 (1998).

[28] O. Biham, E. Milshtein and O. Malcai, Phys. Rev. E 63, 061309 (2001).

[29] C. Tebaldi, M. De Menech and A.L. Stella, Phys. Rev. Lett. 83, 3952 (1999).

[30] S. Lübeck, Phys. Rev. E 61, 204 (2000).

[31] R. Dickman, A. Vespignani and S. Zapperi, Phys. Rev. E 57, 5095 (1998).

[32] R. Dickman, M.A. Munoz, A. Vespignani and S. Zapperi, Brazilian J. Phys 30, 27 (1999).

[33] A. Vespignani, R. Dickman, M.A. Munoz and S. Zapperi, Phys. Rev. E 62, 4564 (2000).

[34] S. Lübeck, Phys. Rev. E 62, 6149 (2000).

[35] M. Paczuski and K.E. Bassler, Phys. Rev. E 62, 5347 (2000).

[36] M. Kloster, S. Maslov and C. Tang, Phys. Rev. E 63, 026111 (2001). 
[37] C.A. Walsh, J.J. Kozak, Phys. Rev. Lett. 47, 1500 (1981).

[38] C.A. Walsh, J.J. Kozak, Phys. Rev. E 26, 4166 (1982).

[39] J.M. Soler, Phys. Rev. B 26, 1067 (1982).

[40] D. Dhar, Physica A 270, 69 (1999).

[41] D. Dhar, P. Ruelle, S. Sen and D.-N .Verma, J. Phys. A 28, 805 (1995).

[42] T. Tsuchiya and M. Katori, Phys. Rev. E 61, 1183 (2000).

[43] D. Dhar, Studying self-organized criticality with exactly solved models, e-print cond-mat/9909009.

[44] E.W.Montroll, in Stochastic Processes in Mathematical Physics and Engineering, edited by Richard Bellman (American Mathematical Society, 190 Hope street, Providence, Rhode Island, 1964), pp. 193-220.

[45] A. Chessa, A Vespignani and S. Zapperi, Comp. Phys. Comm. 121, 299 (1999).

[46] A.N. Kolmogorov, J. Fluid Mech. 13, 82 (1962).

[47] B. Gutenberg and C.F. Richter, Ann. Geophys. 9, 1 (1956).

[48] G. K. Zipf, Humen Behavior and the Principle of least Effort (Addison-Wesley Press, Cambridge, MA, 1949).

[49] D.H. Zanette and S.C. Manrubia, Phys. Rev. Lett. 79, 523 (1997).

[50] V. Pareto, Cours d'Economique Politique, (Macmillan, Paris, 1897), Vol 2 .

[51] B. B. Mandelbrot, 232, 1638 (1951).

[52] B. Mandelbrot, Econometrica 29, 517 (1961).

[53] B. B. Mandelbrot, 36, 394 (1963).

[54] A.-L. Barabasi and R. Albert, Science 286, 509 (1999).

[55] R. Albert and A.-L. Barabasi, Rev. Mod. Phys. 74, 47 (2002).

[56] M. De Menech, A.L. Stella and C. Tebaldi, Phys. Rev. E 58, R2677 (1998).

[57] M. De Menech and A.L. Stella, Phys. Rev. E 62, R4528 (2000). 
FIG. 1: The square lattice of size $3 \times 3$, in which the sites are grouped and indexed according to the symmetry of their positions. The indexing starts from the central site and outwards.

FIG. 2: The average avalanche size for the BTW and Manna models, and the average length of the random-walk paths (starting from random sites) on a finite square lattice with open boundaries, vs. the lattice size $L$. For any value of $L$, it is found that the average avalanche sizes and the average path lengths are all equal.

FIG. 3: Scaling function of the path-length distribution of a random walk on finite lattices of sizes $L=32,64,128,256$ and 512. Here, $n$ is the walker length, $\langle n\rangle_{L}$ is its average and $P(n)_{L}$ is the probability to obtain a path of length $\mathrm{n}$ on a lattice of size $\mathrm{L}$. The five graphs coincide with each other. The slope in the linear range is $-1 / 2$, as expected for the random walk.

FIG. 4: The rescaled avalanche size distributions for the BTW model (a) and for the Manna model (b) for lattice sizes $L=32,64,128,256$ and 512. It is observed that for the BTW model the slope exhibits some dependence on $L$. The slope for best fit obtained by linear regression for lattice size 512 corresponds to $\tau_{L}=1.12 \pm 0.02$ for the BTW model, and $\tau_{L}=1.27 \pm 0.02$ for the Manna model.

FIG. 5: The first three moments of the distribution of the path-lengths of the random walk vs. system size. The filled symbols are the results of direct simulations. The empty symbols are the results of calculations using the Soler method. The slopes of the best linear fits are $1.98 \pm 0.04$, $3.97 \pm 0.06$, and $5.96 \pm 0.08$, for $q=1,2$ and 3 respectively.

FIG. 6: The first three moments of the avalanches sizes in the BTW and the Manna models, vs. system size. The slopes of the best linear fits for the BTW model are $1.98 \pm 0.02,4.68 \pm 0.04$, and $7.52 \pm 0.08$ for $q=1,2$ and 3 respectively. The slopes of the best linear fits for the Manna model are $1.97 \pm 0.02,4.73 \pm 0.04$, and $7.48 \pm 0.08$ for $q=1,2$ and 3 respectively. The results for the first moment are the same for the two models and coincide with the random walk model. The results for higher moments of the two models are not identical, although the differences are small. They both are very different from the random walk results. 


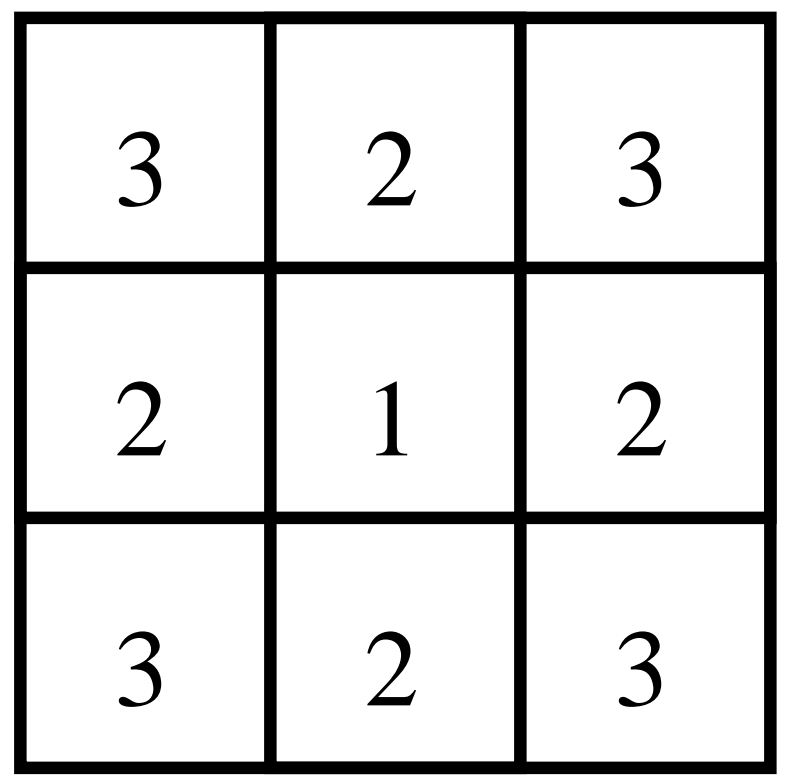

FIG. 1 


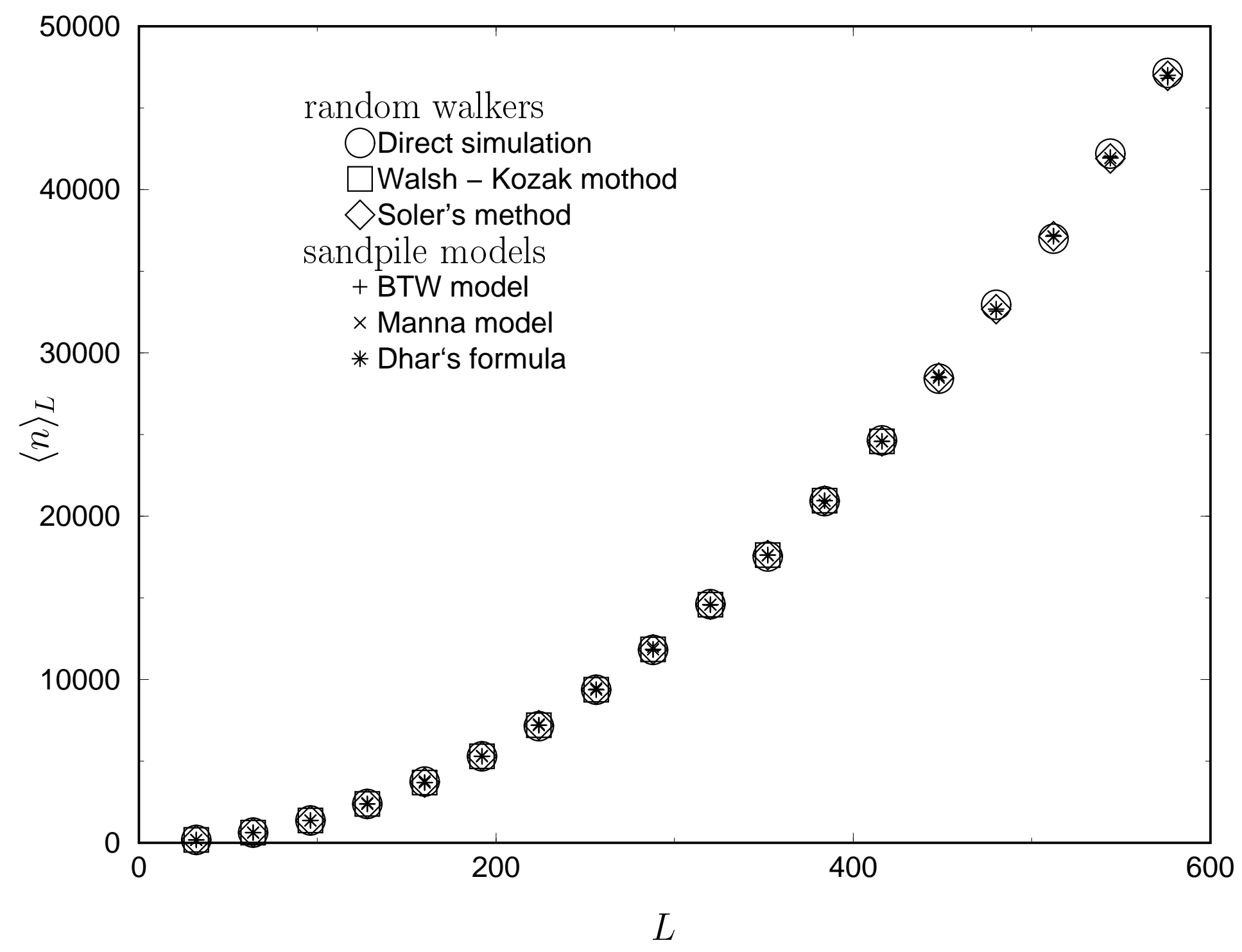

FIG. 2 


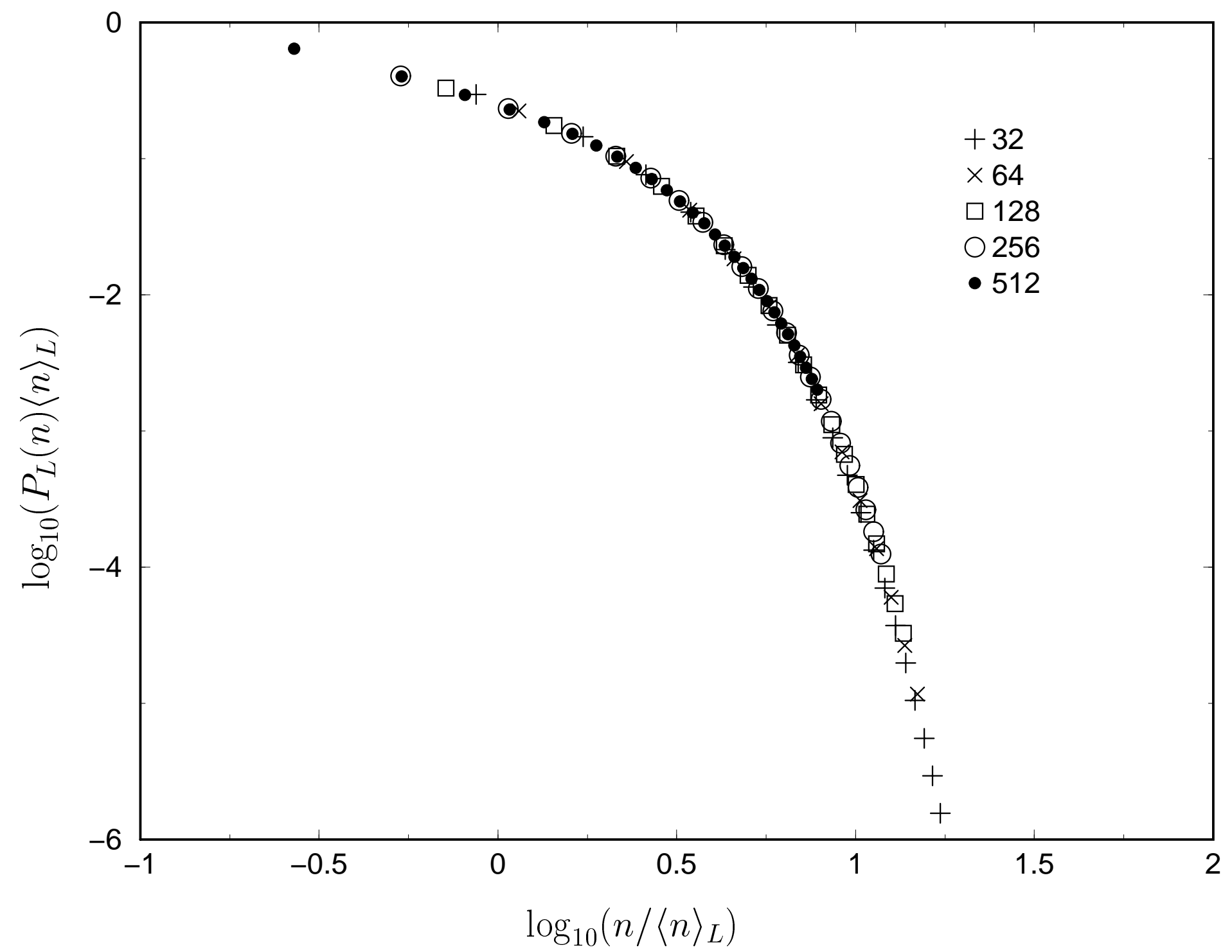

FIG. 3 


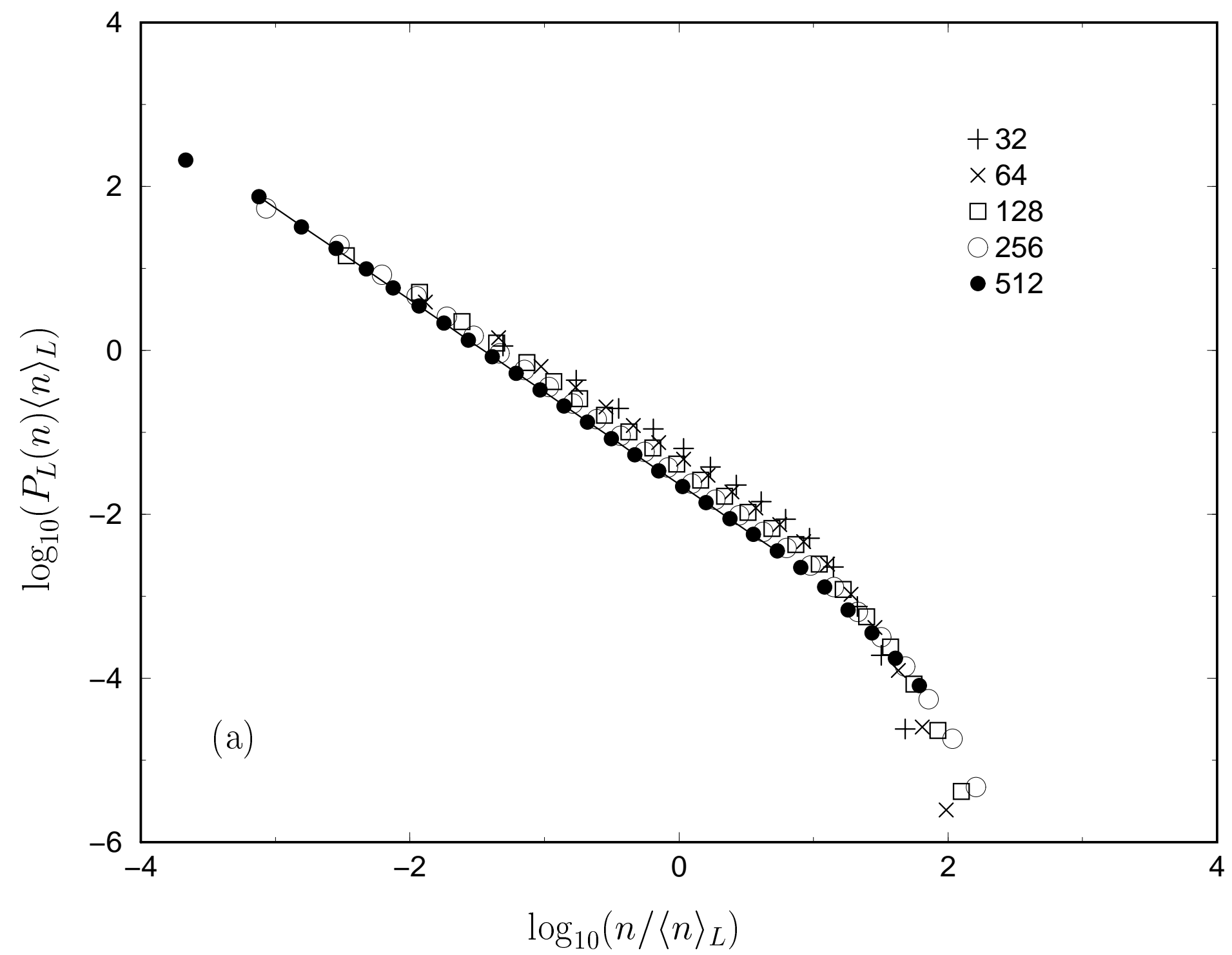

FIG. $4 \mathrm{a}$ 


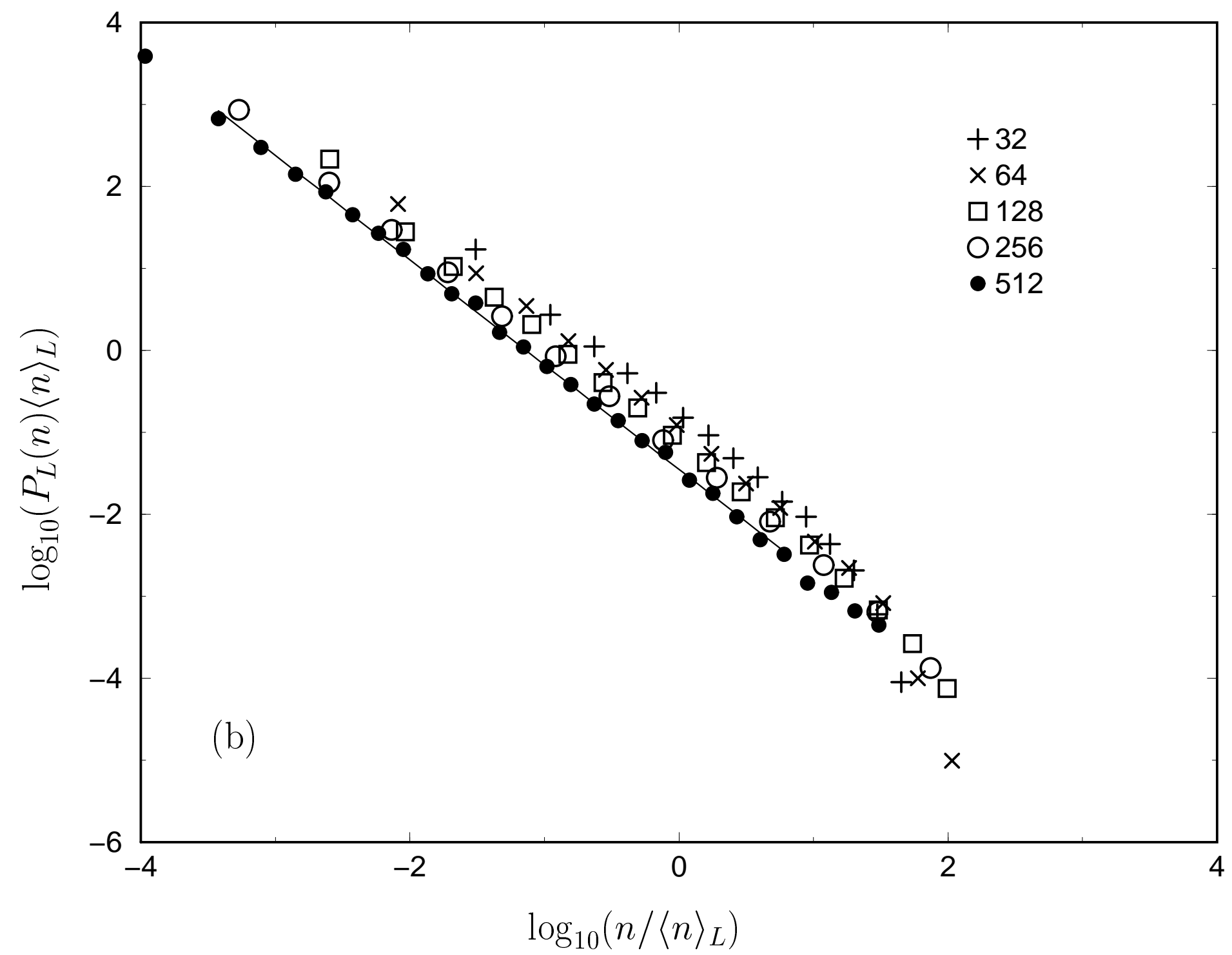

FIG. 4b 


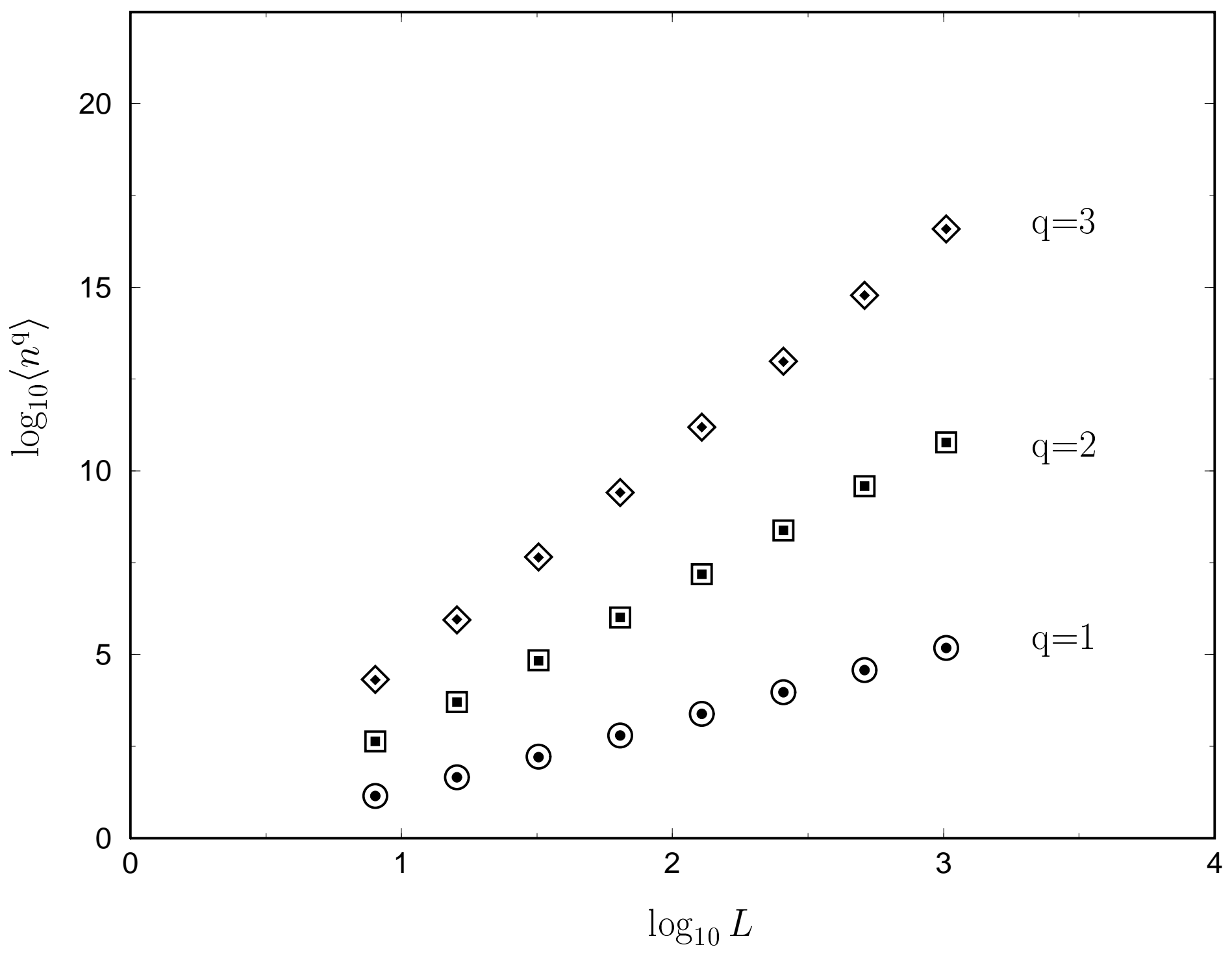

FIG. 5 


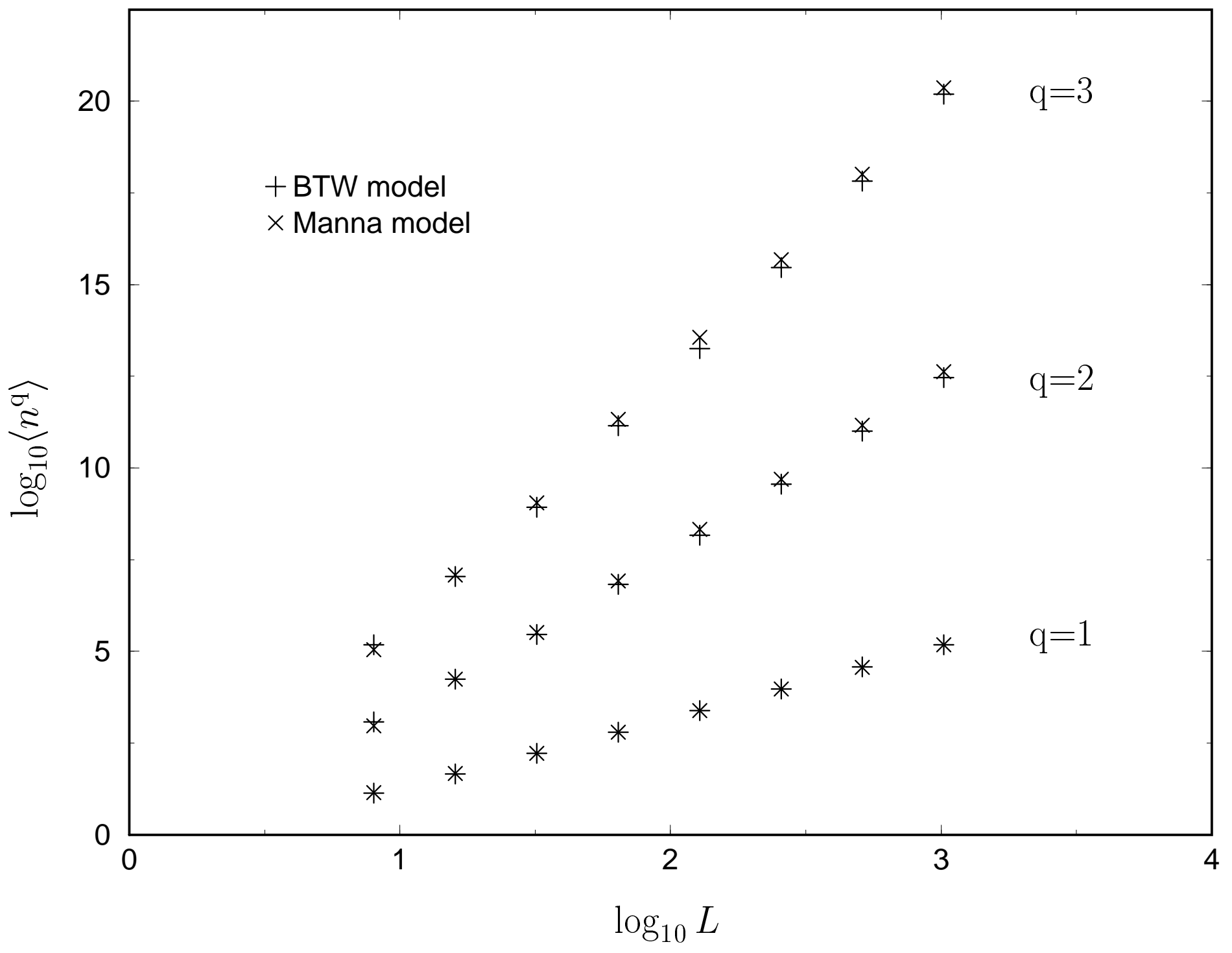

FIG. 6 